\title{
Podocyte Antigens and Glomerular Disease
}

\author{
Pierre Ronco Hanna Debiec \\ INSERM Unit UMR S 702; Pierre et Marie Curie University-Paris 6; AP-HP (Tenon Hospital), Paris, France
}

\section{Key Words}

Membranous nephropathy $\cdot$ Megalin $\cdot$ Neutral

endopeptidase $\cdot$ Alloimmunization $\cdot$ Peptide therapy •

Heymann nephritis

\begin{abstract}
Background: Membranous nephropathy (MN), a major cause of nephrotic syndrome in the adult, is an immune-mediated disease characterized by the accumulation of subepithelial immune deposits leading to complement activation and podocyte injury. However, the target antigens of circulating antibodies are unknown. Current treatments for patients with $\mathrm{MN}$ are entirely empirical, and concept-driven therapies are dramatically lacking. Methods: Specificity of circulating antibodies and composition of glomerular deposits were analyzed in Heymann nephritis (HN), a faithful rat model of $\mathrm{MN}$, and in a subset of patients with antenatal MN. Results: 20 years after the identification of megalin as the podocyte target antigen of nephritogenic antibodies in $\mathrm{HN}$, we identified the human counterpart of megalin, the enzymatic podocyte antigen neutral endopeptidase (NEP). Antibodies to megalin or NEP induce formation of subepithelial immune deposits and of C5b-9, the membrane attack complex of complement. Conclusion: It is likely that antigens involved in idiopathic $\mathrm{MN}$ are expressed at the podocyte membrane. Their identification together with that of immunodominant epitopes may lead to specific antigen/ epitope-based immunotherapy aimed at inducing specific tolerance.

Copyright $\odot 2007$ S. Karger AG, Basel
\end{abstract}

\section{Introduction}

The first podocyte antigen (megalin) involved in glomerular disease was identified in the early 1980s in Heymann nephritis, a rat model of membranous nephropathy (MN) [1]. Twenty years later, a human counterpart to megalin, the neutral endopeptidase (NEP) antigen, was identified in a small subset of patients with antenatal MN [2]. Because the podocyte is continuously exposed to the flux of small amounts of circulating Ig that traverse the glomerular basement membrane (GBM), it is likely that additional podocyte target antigens will be characterized as targets of nephritogenic antibodies, particularly in patients with common forms of idiopathic MN. This nephropathy is characterized by an accumulation of immune deposits (ID) on the outer aspect of the GBM that cause a membrane-like thickening. The ID consist of IgG (mostly IgG4), so far unidentified antigens, and the membrane attack complex of complement C5b-9. As in the experimental model, proteinuria mostly likely results from the formation of subepithelial ID and complement activation leading to podocyte injury.

This review will focus on the long road that has been traveled from the rat to the human $\mathrm{MN}$, and on new mechanisms of immune-mediated glomerular diseases involving podocyte antigens.

\section{KARGER}

Fax +4161306 1234 E-Mail karger@karger.ch www.karger.com
(C) 2007 S. Karger AG, Basel

$1660-2129 / 07 / 1072-0041 \$ 23.50 / 0$

Accessible online at:

www.karger.com/nee
Pierre Ronco

INSERM UMR S 702, Hôpital Tenon

4 , rue de la Chine, FR-75020 Paris (France)

Tel. +3315601 6639, Fax +33156016999

E-Mail pierre.ronco@tnn.aphp.fr 


\section{Podocyte Antigens in Experimental Glomerular Disease: The Case of Heymann Nephritis}

We have learned a great deal about idiopathic MN from Heymann nephritis (HN), which provided the bases of molecular and kinetic concepts of ID formation and glomerular capillary wall injury. The active model of HN is induced by immunization of Lewis rats with preparations of renal brush-border (BB) proteins [1]. Initial studies suggested that the subepithelial ID resulted from glomerular trapping of circulating immune complexes (IC) formed by circulating BB-related antigens and the corresponding antibodies. The development of the model of passive $\mathrm{HN}$ in rats injected with rabbit anti-rat $\mathrm{BB}$ antibodies led to the suggestion that subepithelial ID could be formed without the intervention of circulating IC. Van Damme et al. [3] and Couser et al. [4], using ex vivo and isolated perfused kidney systems, further demonstrated that anti-BB antibodies could bind glomeruli in the absence of circulating BB-related antigen, which provided the proof of principle that IC formation occurred in situ.

The autoantigenic target in the rat disease was identified as the podocyte membrane protein now called megalin $[5,6]$. This antigen is expressed with clathrin at the sole of podocyte foot processes (where IC are formed). The system has been dissected on a molecular level to the precise amino acid sequences of pathogenic epitopes [7, 8]. Megalin is a transmembrane polyspecific receptor protein with a molecular weight of approximately 600 $\mathrm{kDa}$ [9]. This protein is the endocytotic receptor for which the most ligands have been described including receptorassociated protein RAP [9]. This is a $39-\mathrm{kDa}$ protein which acts as a chaperone [9]. Antibodies to RAP were also detected in rats with $\mathrm{HN}$, and passive $\mathrm{HN}$ could be induced by monospecific antibodies against RAP. However, the rats did not develop proteinuria, and ID were cleared within a few weeks from the kidneys of rats injected with anti-RAP antiserum [10]. Furthermore, RAP by itself could not induce active HN. These results indicate a clear divergence in pathogenic potential of megalin and RAP, which may be related to the fact that megalin is an integral membrane protein whereas RAP is not bound to the podocyte membrane.

The HN model also taught us about subsequent steps in the pathogenesis of MN. Complement activation by the podocyte-associated megalin-anti-megalin IC is required for proteinuria to occur [11, 12]. Kerjaschki et al. [13] directly visualized the insertion of C5b-9 into the podocyte plasma membrane and its transcellular trans-

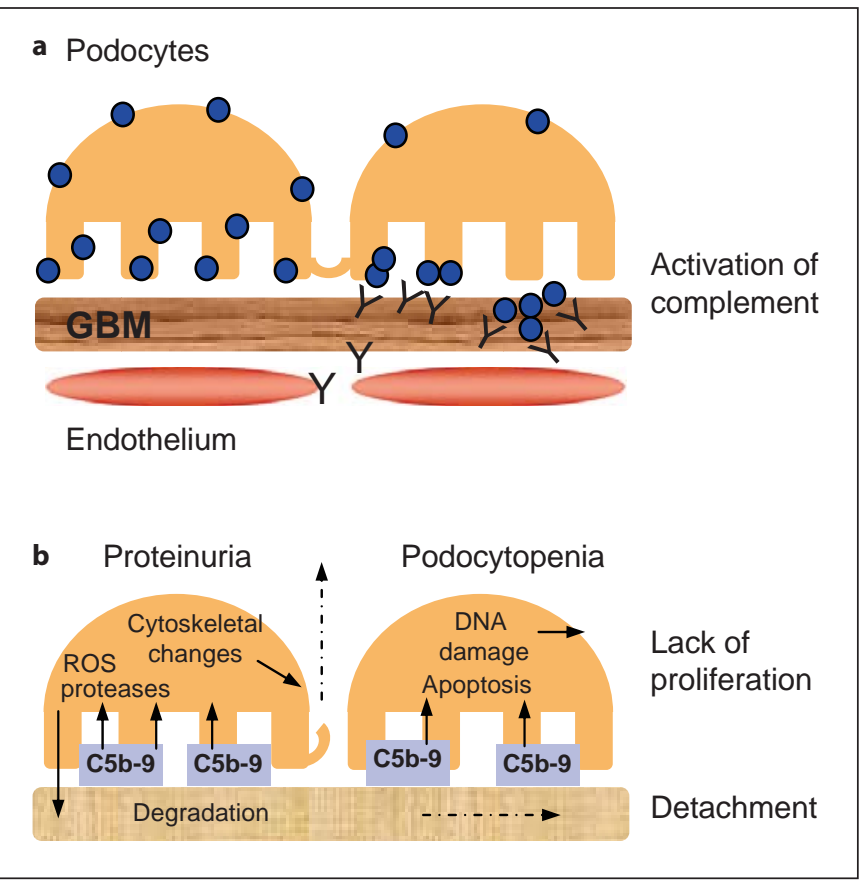

Fig. 1. a In situ formation of immune deposits in neonatal MN. NEP (blue dots) serves as pathogenic antigen in the podocyte's cell membrane. Antibodies to this protein originate in women who lack NEP epitopes because of truncating mutations. Antiendopeptidase antibody is transported across the placenta and causes the formation of immune complexes at podocyte membranes, similar to those observed in experimental HN. It is likely that as for megalin (the antigen of HN), NEP-anti-NEP immune complexes are then shed and rapidly immobilized in the GBM, thus preventing clearance of the complexes by the podocyte through endocytosis. b Schematic description of the cellular mechanisms that lead to proteinuria in MN. C5b-9 formation on the membrane of podocytes leads to various intracellular events, including production of reactive oxygen species (ROS) and proteases, and cytoskeletal changes. These result in degradation of GBM and redistribution of proteins that compose the slit diaphragm, eventually leading to the development of protein leakage into the Bowman's space (left). In addition, C5b-9 attack leads to podocytopenia through apoptosis, lack of proliferation resulting from complement-induced DNA damage, and podocyte detachment (right).

port by podocytes. Membrane insertion of C5b-9 leads to a cascade of cellular events that dramatically affect the functions of podocytes and the glomerular capillary wall (see fig. 1 for NEP antigen).

Megalin cannot be responsible for human MN because it has not been found in human glomeruli or podocytes, nor has it been detected in subepithelial ID in patients with MN. Other podocyte membrane proteins such as dipeptidyl peptidase IV (DPPIV) [14], NEP [15] and 
aminopeptidase A [16] were shown to serve as target antigens for circulating antibodies in rats, rabbit and mice, respectively. Because both DPPIV and NEP are expressed on the human podocyte, we hypothesized some 18 years ago that these two enzymatic antigens might play some role in the pathogenesis of $\mathrm{MN}$ in humans [15].

\section{NEP, the First Podocyte Target Antigen Identified in Human MN}

From 2002 to 2004, we identified in three families a human counterpart to $\mathrm{HN}$ in infants born with nephrotic syndrome, acute renal failure or both, and histologically diagnosed $\mathrm{MN}[2,17]$. We found that pathogenic antibodies directed against NEP were transplacentally transferred from the mother to her child. NEP is a membrane-bound enzyme that can digest biologically active peptides. It is expressed on the surface of human podocytes and syncytiotrophoblastic cells, as well as on polymorphonuclear leukocytes, lymphoid progenitor cells, and epithelial cells of non-lymphoid organs. The anti-NEP antibodies produced by the mother, which were transiently found in the infant's serum, were most likely responsible for the infant's $\mathrm{MN}$, given that the injection of rabbits with the serum IgG fraction from the mother induced intraglomerular deposits and proteinuria, whereas injection with the IgG fraction from the father did not. Furthermore, NEP was localized by confocal microscopy in ID together with C5b-9, both in the infant and in rabbits that received an injection with the mother's IgG [18].

The 4 cases of antenatal MN because of transplacental transfer of anti-NEP antibodies led us to revisit the concept of in-situ-formed compared with circulating-preformed IC, which has been debated for the past 30-40 years. It is most likely that in these cases, IC were predominantly formed in situ at the sole of podocyte foot process where NEP is expressed (fig. 1a). NEP is found in a diffuse pattern on the membrane of podocytes, as is angiotensin-converting enzyme on the plasma membrane of mature oocytes [19]. In-vivo interaction of angiotensin-converting enzyme with divalent antibodies induces the formation of granular ID through a mechanism of 'patching' and 'shedding' of IC [19]. A similar mechanism may be implicated in the formation of ID in the infants' glomeruli between the lamina rara externa and the podocytes' slit diaphragms. Transient low levels of circulating IC that contained NEP were detected in the infant's serum [2]. Their contribution to the formation of subepithelial ID is uncertain, because levels of circulating IC were low, manifestations of serum sickness were absent, and subendothelial and mesangial ID were not seen. The two mechanisms of IC formation (in situ compared with preformed) are not mutually exclusive.

\section{From NEP-Induced Antenatal MN to Alloimmunization}

\section{Antenatal MN as a Model of Alloimmune Glomerular} Disease in the Native Kidney

As the children's mothers were healthy with normal renal function (although with high titers of circulating anti-NEP antibodies), we proposed that these women were NEP-deficient. This hypothesis was confirmed by our genetic studies that identified two truncating mutations in the $M M E$ gene coding for NEP. We could not detect truncated proteins in the NEP-deficient mothers' granulocytes or urine (sites of NEP abundance under normal circumstances), or in their heterozygous children, in whom only normal NEP was detected [17]. These findings indicate that the mutated $M M E$ gene is knocked out functionally, probably a result of instability of the mutated messenger RNA or protein.

We have thus characterized a novel fetomaternal disease in which a genetic defect of the mother causes development of MN in her fetus. Currently, rhesus incompatibility is the paradigm of fetomaternal diseases due to alloimmunization, and only diseases of this type that affect red blood cells and platelets have been described. Our findings bring to light the possibility that truncating mutations of other podocyte antigens, which do not cause symptoms in the carrier mother, lead to alloimmunization of the fetus following transplacental movement of nephritogenic antibodies. Similarly, immunization against allovariants of proteins expressed by placental cells in the mother and by glomerular cells in the fetus might cause neonatal renal disease.

\section{Podocyte Target Antigens in the Renal Graft}

When end-stage renal failure is caused by a genetic podocyte defect, there is a risk of a specific alloimmune adverse reaction if the patient - who lacks a specific antigen - is transplanted with a normal donor kidney that harbors the antigen [20]. In addition to a Goodpasture'slike syndrome occurring after transplantation in patients with Alport's syndrome, the risk of alloimmunization is confirmed by observations in patients with congenital nephrotic syndrome of the Finnish type (CNF) caused by mutations of NPHS1 that encodes nephrin, a key protein 
of the podocyte slit diaphragm. Proteinuria recurs in about $25 \%$ of transplanted patients with CNF [21] almost exclusively in those of the Fin-major/Fin-major genotype characterized by complete absence of nephrin in the native kidney. Of 9 patients with recurrent nephrotic syndrome, 8 harbored glomerulus-reactive antibodies, and 4 had high levels of anti-nephrin antibodies [21]. It is likely that anti-nephrin antibodies have a role in the development of severe proteinuria, as in experimental animal models [22]. This hypothesis is supported by the efficacy of rescue therapy with cyclophosphamide.

The discovery of alloimmune neonatal $\mathrm{MN}$ induced by anti-NEP antibodies might also shed new light on the pathogenesis of de novo MN which develops after renal transplantation. Analogies can be drawn between the pregnant mother and the graft recipient on the one hand, and the fetus (and the placenta) and kidney donor on the other. Because NEP deficiency is asymptomatic in humans, NEP-deficient graft recipients are not identified prior to transplant. These individuals are most likely to raise an anti-NEP alloimmune response when their immune system is exposed to NEP in the donor kidney.

\section{Alloimmune Nephropathies in the Native Kidney:}

Do They Occur in the Adult?

The view that alloimmune nephropathies may occur in adult native kidneys is supported by observations made in patients receiving a bone marrow transplant or allogeneic blood stem cells, especially in those suffering graftversus-host disease [20]. MN is by far the most common histologic lesion, accounting for more than $30 \%$ of cases [23]. Podocyte-associated proteins might serve as targets for the circulating alloimmune antibodies that are directed against a podocyte antigen expressed in the recipient but absent from the donor, or against an allovariant. Autoantibodies against nephrin were produced in an experimental model [24]. The possible connection between fetal cell microchimerism (i.e. long-term persistence of fetal cells in the mother) and $\mathrm{MN}$ is worthy of investigation.

\section{Involvement of Podocyte Antigens in 'Autoimmune' MN: Still a Matter of Debate}

It is unlikely that the subepithelial deposits characteristic of $\mathrm{MN}$ are the consequence of glomerular trapping of preformed IC directly from the circulation. However, such deposits can be produced by local or in situ IC for- mation involving antigens that could be exogenous or endogenous and could act in three ways. First, exogenous antigens could localize on the subepithelial surface because of their cationic charge and small size. Second, they could form IC on the inner (endothelial) surface of the capillary wall that then dissociate, traverse the GBM and re-form in the subepithelial space. In so-called secondary forms of MN, hepatitis B, hepatitis C and Helicobacter pylori antigens, tumor antigens and thyroglobulin have been detected in subepithelial deposits, but there is no real proof that these antigens are pathogenic [18]. They may simply have been trapped passively between the lamina rara externa and the slit diaphragm because of increased permeability of the glomerular capillary wall to proteins, as is the case for albumin.

In the third scenario, endogenous constituents of the glomerular capillary wall, mostly antigens of the podocyte membrane, serve as targets for autoimmune antibodies. This scenario is supported by the total absence of deposits at subendothelial (and mesangial) sites in idiopathic $\mathrm{MN}$, as well as the ability to induce the disease with anti-podocyte antibodies including anti-megalin in the rat and anti-NEP in humans. As yet, we have failed to detect NEP in the ID in patients with MN. All past studies performed with sera or Ig eluted from patients with MN have failed to identify relevant antigens. The nephritogenic antibodies most often circulate in very low amount and/or may be diluted in kidney Ig eluates from aged $\mathrm{MN}$, so we have developed sensitive methods to detect the target antigens of circulating antibodies in patients with MN. Some of those antigens have been identified by mass spectrometry. Most of these proteins are not specific for podocytes, which suggests that the corresponding antibodies might recognize a neo-epitope on podocyte antigens that fails to be expressed on other cell types.

\section{Towards Antigen (Epitope)-Driven Therapies in Patients with MN}

Current treatments for patients with $\mathrm{MN}$ are entirely empirical: concept-driven therapies are dramatically lacking. The design of specific therapies for autoimmune diseases is primarily based on induction of specific immune tolerance. Ideally, this requires identification of pathogenic epitopes borne by the antigen. One way to induce tolerance is mucosal administration of antigen or immunodominant synthetic peptides. Nasal administration of recombinant $\mathrm{NC} 1$ domain of the $\alpha 3$ chain of type 
IV collagen was shown to induce tolerance in a model of anti-GBM glomerulonephritis [25].

We have recently identified two immunodominant epitopes in the NEP antigen that are specifically recognized by the mothers' antibodies [26]. Future pregnancies in NEP-immunized mothers are at high risk for the fetus [27] so that epitope-driven therapies including induction of mucosal tolerance may be needed in addition to nonspecific immunosuppressive therapy based on intravenous Ig and high-dose corticosteroids. A similar approach could be used in idiopathic $\mathrm{MN}$ once the target (podocyte) antigen is identified. In patients with immunologically active glomerular disease, a combination of non-specific and antigen/epitope-driven therapies should be envisaged. For instance, the effect of anti-CD20 monoclonal antibodies on Ig production could be complemented by peptide-based immunotherapy aimed at reducing specifically the synthesis of anti-podocyte antibody.

In conclusion, substantial progress has been made in the past few years in understanding the pathophysiology of human MN. The first human podocyte antigen has been identified. Anti-NEP antibodies do not cause idiopathic $M N$, but the experimental and human data strongly suggest that most antigenic targets sit at the podocyte membrane, providing a focus for the search for other novel antigens. Translational research in this area should soon lead to assays of circulating pathogenic antibodies and to better targeted therapy aimed at decreasing specifically their production.

\section{Acknowledgements}

The research of the authors is funded by grants from GIS-Institut des Maladies Rares, INSERM, AURA (Association pour l'Utilisation du Rein Artificiel), Programme Hospitalier de Recherche Clinique (Vincent Guigonis), Agence de la Biomédecine, AMGEN, and Genzyme Renal Innovations Program (GRIP). We are indebted to Béatrice Mougenot (Tenon Hospital, Paris) and Vincent Guigonis (Limoges University Hospital) for precious collaboration. We thank Christine Vial for assistance in editing the manuscript.

\section{References}

1 Heymann W, Hackel DB, Harwood S, Wilson SGF, Hunter JLP: Production of nephrotic syndrome in rats by Freund's adjuvants and rat kidney suspension. Proc Soc Exp Biol Med 1959;100:660-664.

-2 Debiec H, Guigonis V, Mougenot B, Decobert F, et al: Antenatal membranous glomerulonephritis due to anti-neutral endopeptidase antibodies. N Engl J Med 2002;346: 2053-2060.

-3 Van Damme BJ, Fleuren GJ, Bakker WW, Vernier RL, Hoedemaeker PJ: Experimental glomerulonephritis in the rat induced by antibodies directed against tubular antigens. V. Fixed glomerular antigens in the pathogenesis of heterologous immune complex glomerulonephritis. Lab Invest 1978;38:502510.

-4 Couser WG, Steinmuller DR, Stilmant MM, Salant DJ, Lowenstein LM: Experimental glomerulonephritis in the isolated perfused rat kidney. J Clin Invest 1978;62:1275-1287.

-5 Kerjaschki D, Farquhar MG: The pathogenic antigen of Heymann nephritis is a membrane glycoprotein of the renal proximal tubule brush border. Proc Natl Acad Sci USA 1982;79:5557-5561.

6 Kerjaschki D, Farquhar MG: Immunocytochemical localization of the Heymann nephritis antigen (gp330) in glomerular epithelial cells of normal Lewis rats. J Exp Med 1983;157:667-686.
7 Tramontano A, Knight T, Vizzuso D, Makker SP: Nested N-terminal megalin fragments induce high-titer autoantibody and attenuated Heymann nephritis. J Am Soc Nephrol 2006;17:1979-1985.

8 Ronco P, Debiec H: Molecular dissection of target antigens and nephritogenic antibodies in membranous nephropathy: towards epitope-driven therapies. J Am Soc Nephrol 2006; 17:1772-1774.

-9 Christensen EI, Birn H: Megalin and cubilin: multifunctional endocytic receptors. Nat Rev Mol Cell Biol 2002;3:256-266.

10 Makker SP, Tramontano A: Differential capacity of anti-RAP and anti-megalin antibodies to produce progressive passive Heymann nephritis - implications for the pathogenesis of idiopathic human membranous glomerulonephritis. J Pathol 2006;210: 282-287.

11 Salant DJ, Belok S, Madaio MP, Couser WG: A new role for complement in experimental membranous nephropathy in rats. J Clin Invest 1980;66:1339-1350.

12 Cybulsky AV, Rennke HG, Feintzeig ID, Salant DJ: Complement-induced glomerular epithelial cell injury. Role of the membrane attack complex in rat membranous nephropathy. J Clin Invest 1986;77:1096-1107.
13 Kerjaschki D, Schulze M, Binder S, Kain R, Ojha PP, Susani M, Horvat R, Baker PJ, Couser WG: Transcellular transport and membrane insertion of the C5b-9 membrane attack complex of complement by glomerular epithelial cells in experimental membranous nephropathy. J Immunol 1989;143: 546-552.

14 Ronco P, Allegri L, Melcion C, Pirotsky E, et al: A monoclonal antibody to brush border and passive Heymann nephritis. Clin Exp Immunol 1984;55:319-332.

$\checkmark 15$ Ronco P, Allegri L, Brianti E, Chatelet F, et al: Antigenic targets in epimembranous glomerulonephritis. Experimental data and potential application in human pathology. Appl Pathol 1989;7:85-98.

16 Assmann KJ, van Son JP, Dijkman HB, Koene RA: A nephritogenic rat monoclonal antibody to mouse aminopeptidase A. Induction of massive albuminuria after a single intravenous injection. J Exp Med 1992;175: 623-635.

$\checkmark 17$ Debiec H, Nauta J, Coulet F, van der Burg M, et al: Role of Truncating mutations in MME gene in feto-maternal allo-immunization and neonatal glomerulopathies. Lancet 2004;364:1252-1259.

18 Ronco P, Debiec H: Molecular pathomechanisms of membranous nephropathy: from Heymann nephritis to alloimmunization. J Am Soc Nephrol 2005;16:1205-1213. 
19 Matsuo S, Caldwell PRB, Brentjens JR, Andres G: In vivo interactions of antibodies with cell surface antigens. A mechanism responsible for in situ formation of immune deposits in the zona pellucida of rabbit oocytes. J Clin Invest 1985;75:1369-1380.

-20 Ronco P, Debiec H, Guigonis V: Mechanisms of disease: alloimmunization in renal diseases. Nat Clin Pract Nephrol 2006;2:388-397.

-21 Patrakka J, Ruotsalainen V, Reponen P, Qvist E, Laine J, Holmberg C, Tryggvason K, Jalanko H: Recurrence of nephrotic syndrome in kidney grafts of patients with congenital nephrotic syndrome of the Finnish type: role of nephrin. Transplantation 2002; 73:394-403.
-22 Topham PS, Kawachi H, Haydar SA, Chugh S, Addona TA, Charron KB, Holzman LB, Shia M, Shimizu F, Salant DJ: Nephritogenic mAb 5-1-6 is directed at the extracellular domain of rat nephrin. J Clin Invest 1999;104: 1559-1566.

23 Ikee R, Yamamoto K, Kushiyama T, Imakiire T, Suzuki S, Miura S: Recurrent nephrotic syndrome associated with graft-versus-host disease. Bone Marrow Transplant 2004;34: 1005-1006.

24 Nagahama K, Maru K, Kanzaki S, Chai HL, Nakai T, Miura S, Yamaguchi A, Yamanaka S, Nagashima Y, Aoki I: Possible role of autoantibodies against nephrin in an experimental model of chronic graft-versus-host disease. Clin Exp Immunol 2005;141:215-222.

$\checkmark 25$ Reynolds J, Prodromidi EI, Juggapah JK, Abbott DS, Holthaus KA, Kalluri R, Pusey CD: Nasal administration of recombinant rat $\alpha 3$ (IV)NC1 prevents the development of experimental autoimmune glomerulonephritis in the WKY rat. J Am Soc Nephrol 2005; $16: 1350-1359$
26 Debiec H, Luimula P, Lefeu F, Nortier JL, Ronco P: Identification of B-cell epitopes on neutral endopeptidase in feto-maternal alloimmunization with antenatal glomerulopathies (abstract). J Am Soc Nephrol 2006;17: F-PO065.

27 Nortier JL, Debiec H, Tournay Y, Mougenot B, Noël JC, Deschodt-Lanckman MM, Janssen F, Ronco P: Neonatal disease in neutral endopeptidase alloimmunization: lessons for pregnancy management and immunological monitoring. Pediatr Nephrol 2006; 21:1399-1405. 